\title{
Suicide and attempted suicide
}

\author{
SS Williams, T Rajapakse
}

\section{The neurobiology of suicide (1)}

Accurate prediction of suicide risk remains a challenge, in its prevention. Many people with psychiatric disorders do not manifest suicidal behaviour, suggesting the importance of diathesis, or susceptibility to suicide, in addition to associated disorders. At present there are no biomarkers to predict suicidal behaviour. The authors of this article have attempted to describe the neurobiology of suicide. To do so, they have reviewed the literature from 1990 onwards, using the keywords suicide, neurobiology, genetics and neuroimaging. Based on their findings, the authors go on to discuss the stress-diathesis model of suicide, which posits that suicide is a result of interactions between state-dependent (environmental) stressors and trait-like diathesis (or susceptibility to suicidal behaviour), independent of psychiatric disorders.

Post-mortem findings from people who have died by suicide (such as increased number of serotonin neurons in the brain stem, reduced noradrenergic neurons in the locus coeruleus, and reduced volume of the dentate gyrus), and findings of functional neuroimaging studies (such as hypo-functioning of the medial prefrontal and lateral prefrontal cortex being associated with increased lethality of suicide attempts) are cited as evidence of the trait-like diathesis or susceptibility to suicidal behaviour. The authors argue that up to $50 \%$ of suicidal behaviour is heritable; and that genetic factors may be involved in changes in brain circuitry, as well as volume changes of the brain - for example in the fronto-striatal areas of the brain.

Stressors such as life-events (e.g., childhood sexual abuse) and psychiatric disorders are important risk factors for suicide; however, the authors postulate that the diathesis concept explains why, only a few of the individuals who are exposed to such stressors go on to take their own life. The experience of repeated abuse, particularly physical and sexual abuse, is a reported environmental stressor that increases susceptibility to suicide. The authors suggest this may be associated with epigenetic modifications of specific neurotransmitter systems and brain circuitry involved in mood regulation. Changes of neuronal circuitry, probably mediated via noradrenergic transmission and the hypothalamicpituitary-adrenal axis, are postulated. Details of how this occurs are not known, but it is thought that it may involve DNA methylation of glucocorticoid receptors in the brain. At present, clinical predictors of suicide risk have limited effectiveness. Based on the stress-diathesis model, the authors argue that genomics and brain imaging may predict new directions for prediction of suicide. The authors also postulate novel methods for suicide prevention, such as repetitive transcranial magnetic stimulation to modify functional activity in the orbitofrontal cortex, and novel psychopharmacological compounds, such as drugs that target the hypothalamic-pituitaryadrenal axis. This article offers new insights into possible future directions of suicide prediction and prevention, but more research is needed to further explore these hypotheses.

\section{Newspaper coverage of suicide and initiation of suicide clusters in teenagers in the USA, 1988-96: a retrospective, population-based case-control study (2)}

The term suicide 'cluster' refers to an excessive number of suicides occurring in close temporal and geographic proximity, and approximates the concept of an 'outbreak' of suicide in a particular community. The aim of this study was to examine whether an environmental factor newspaper reports of suicide - has a role in the emergence of suicide clusters.

This was a retrospective, population based, case-control study. Suicide clusters from 1988-1996, among young people aged 13-20 years in the US, were identified using the time-space scan statistic. Each identified cluster community was matched with two non-cluster control communities (in which suicides of similarly aged youths occurred, but without clustering). Thereafter newspapers published in the cluster community, between the first and second suicide in that cluster, were examined for stories about suicide. In the control communities, newspapers were examined for a similar matched level of time after the control suicide. Content-analysis procedure was used to code characteristics of each newspaper story, and mixed-effect regression was used to compare the case and control communities.

For the period of the study, 53 suicide clusters were identified. When case and control communities were compared, the mean number of news stories about suicidal individuals published after an index cluster suicide was significantly greater (7.42, SD 10.02) than the mean number of suicide stories published after a non-cluster suicide (5.14, SD 6.00; $<<.0001$ ). Several key story features, such as front-page placement, headlines containing the word 'suicide' or a description of the 
method used, and detailed descriptions of the suicidal individual and act, were reported more often in the stories published after the index cluster suicides than after the non-cluster suicides.

This study provides interesting insights into how newspaper reporting may contribute towards clustering of suicides among young people. Although derived from a study in the US, the findings have implications of other countries and this area is worth examining with further research in Sri Lanka.

\section{Attempted suicide in bipolar disorder: risk factors in a cohort of 6086 patients (3)}

Bipolar affective disorder is associated with an increased risk of suicidal behaviour. The authors of this study attempted to examine risk factors for attempted suicide in bipolar disorder, using a large patient sample to allow for comparison of severity of risk factors.

Data was derived from a Swedish national register, which contains detailed individual data for patients diagnosed with bipolar type 1, type 2, and schizoaffective disorder of bipolar type, and which is maintained by psychiatrists and especially staff trained staff. The study population consisted of 6086 bipolar patients, who were followed up annually between 2005-2012. The mean total followup time was 2.4 years $(\mathrm{SD}=1.3)$. The cohort consisted of 2408 men and 3678 women, with a mean age of 49.3 years $(\mathrm{SD}=12.8)$ and $48.3(\mathrm{SD}=13.0)$ years respectively. For the purposes of this study, the authors defined attempted suicide as per the ICD-10 category of intentional selfharm (codes X60-84).

In this cohort, 13 suicides and 338 attempted suicide occurred during the follow-up period. Attempted suicide during follow-up was significantly more likely in women (6.9\%) compared to men (4.1\%; $\left.\chi^{2} 20.61, \mathrm{df}=1, \mathrm{p}<.001\right)$. Multiple regression analysis showed that previous suicide attempts at baseline (men: $\mathrm{OR}=3.93,95 \% \mathrm{CI}=2.48$ 6.24; women: $\mathrm{OR}=4.24$, 95\% $\mathrm{CI}=3.06-5.88)$, affective episodes during the year before baseline (men: $\mathrm{OR}=3.63$, 95\% CI=1.76-7.51; women: $\mathrm{OR}=2.81$, 95\% CI=1.78-4.44), a history of many lifetime depressive episodes (men: $\mathrm{OR}=2.06,95 \% \mathrm{CI}=1.08-3.92$; women: $\mathrm{OR}=1.93$, 95\% $\mathrm{CI}=1.27-2.94)$ and psychiatric inpatient care during the year before baseline (men: $\mathrm{OR}=3.57,95 \% \mathrm{CI}=1.59-8.01$; women: $\mathrm{OR}=2.68,95 \% \mathrm{CI}=1.60-4.50$ ), predicted suicide attempts during follow-up. Gender differences also emerged. Comorbid substance use disorder was a predictor in men $(\mathrm{OR}=1.95,95 \% \mathrm{CI}=1.11-3.44)$, while comorbid personality disorder was a predictor in women $(\mathrm{OR}=2.29,95 \% \mathrm{CI}=1.42-3.69)$.

This is a large study which indicates that those with more severe and unstable forms of bipolar disorder, in terms of having had hospital admissions in the past year, past suicide attempts and a life-time history of more depressive episodes, are more likely to attempt suicide. The authors also suggest that there maybe differing risk factors for men and women with bipolar disorder, which requires further research.

\section{Differences in risk factors for self-harm with and without suicidal intent: findings from the ALSPAC cohort (4)}

Despite increased awareness of the importance of selfharm, the manner in which this phenomenon should be conceptualised is still debated. Some researchers argue that a clear distinction can be made between acts of self harm that occur with intent to die (suicide attempts, SA), and those that occur with no intent to die (Non-Suicidal Self-Injury NSSI). However, many individuals engage in both behaviours, leading some researchers to argue that these phenomena are best conceptualised along a continuum.

The objective of this study was to compare self-harm with and without suicidal intent, among adolescents, to determine differences (if any) in associated risk factors. The authors used data from the Avon Longitudinal Study of Parents and Children (ALSPAC), an ongoing population-based birth cohort study examining influences on health and development across the life course. Information was derived based on 4799 adolescents who completed a detailed self-harm questionnaire at age 16 years. Participants who responded positively to the item "have you ever hurt yourself on purpose in any way (e.g., by taking an overdose of pills or by cutting yourself?)"' were classified as having a history of self-harm. Additional questions were used to determine those who had self-harmed with suicidal intent.

Of the 4799 participants under consideration, 11.9\% $(n=569)$ reported self-harm without suicidal intent, but no episodes of suicidal self harm; $6.8 \%(n=325)$ reported self-harming with suicidal intent on at least one occasion. When considered overall, females were more likely to report self-harm than males, and the self-harming groups had higher levels of risk factors compared to those with no history of self-harm.

When comparing risk factors associated with self-harm with suicidal intent versus without suicidal intent, results showed both similarities and differences. Female gender, childhood sexual abuse, heavy drinking, cannabis use and higher intensity seeking scores were strongly associated both with self-harm with suicidal intent, as well as self-harm without suicidal intent. Mental health problems such as depression and anxiety disorders were also associated with both types of self-harm, but the association was stronger for self-harm with suicidal intent. Higher novelty-seeking scores were associated more with self-harm without suicidal intent, compared to self-harm with suicidal intent. 
When considering specific risk factors, lower socioeconomic status, cruelty to children in the household, parent suicide attempts and paternal self-harm were risk factors for self-harm with suicidal intent. On the other hand, higher IQ and maternal education increased the risk of self-harm without suicidal intent, while it decreased the risk of self-harm with suicidal intent.

Limitations of this study include a possible selection bias, factors that may have influenced reporting of suicide intent (such as participant mood state at time of assessment) and the fact that the study did not distinguish between participants with a lifetime history of self-harm with suicidal intent, and those with both self-harm with suicidal intent plus self-harm without suicidal intent.

The overall findings of the study indicate both common and specific risk factors for self-harm with and without suicidal intent. The authors suggest that self-harm with and without suicidal intent could be distinguished in terms of their relationship with a number of recognised risk factors, which suggest that these are overlapping behaviours, but with some distinct characteristics as well. Further research is needed to investigate whether these behaviours have different clinical outcomes, and to guide future assessment and management.

From pesticides to medicinal drugs: time series analyses of methods of self-harm in Sri Lanka (5)

Sri Lanka's suicide rates have shown a steady decline since the mid 1990s, when it was at its highest. The authors attempt to explore if recent changes in methods of self-harm in Sri Lanka could explain the decline in the incidence of suicide.

A time series analyses of suicide rates and hospitalization due to different types of poisoning was carried out.

The authors report that between 1996 and 2008 the annual incidence of hospital admission due to poisoning by medicinal or biological substances increased exponentially, from 48.2 to 115.4 admissions per 100,000 population. Over the same period, annual admissions resulting from poisoning with pesticides decreased from
105.1 to 88.9 per 100,000 . The annual incidence of suicide decreased exponentially, from a peak of 47.0 per 100,000 in 1995 to 19.6 per 100000 in 2009. Poisoning accounted for 37.4 suicides per 100,000 population in 1995 but only 11.2 suicides per 100,000 in 2009 . The case fatality rate for pesticide poisoning decreased linearly, from 11.0 deaths per 100 cases admitted to hospital in 1997 to 5.1 per 100 in 2008.

The study concludes that since the mid 1990s, a trend away from the misuse of pesticides (despite no reduction in the general availability of pesticides) and towards increased use of medicinal and other substances has been seen in Sri Lanka, among those seeking self-harm. These trends, together with the reduction of mortality among those suffering pesticide poisoning, are opined to have resulted in the overall reduction in the national incidence of completed suicide.

\section{Declaration of interest}

None declared

Shehan Williams, Faculty of Medicine University of Kelaniya, Sri Lanka

Thilini Rajapakse, Faculty of Medicine, University of Peradeniya, Sri Lanka

\section{References}

1. Heeringen KV, Mann JJ. The neurobiology of suicide. Lancet Psychiatry 2014; 1: 63-72.

2. Gould MS, Kleinman MJ, Lake AM, Forman J, Midle JB. Lancet Psychiatry 2014; 1(1): 34-43.

3. Tidemalm D, Haglund A, Karanti A, Landen M, Runeson B. Attempted suicide in bipolar disorder: risk factors in a cohort of 6086 patients. PLOS one 2014; DOI: 10.1371/ journal. pone. 0094097.

4. Mars B, Heron J, Crane C, et al. Differences in risk factors for self-harm with and without suicidal intent: findings from the ALSPAC cohort. J Affect Dis 2014; 168: 407-14.

5. de Silva VA, Senanayake SM, Dias P, Hanwella R. From pesticides to medicinal drugs: time series analyses of methods of self-harm in Sri Lanka. Bull World Health Organ 2012; 90(1): 40-6. 